\title{
Radar Detection of High-Energy Cosmic Rays in non-Gaussian Background Using a Time-Frequency Technique
}

\author{
Mohamed Abou Bakr Othman ${ }^{\mathrm{a}}$, John Belz ${ }^{\mathrm{b}}$, Behrouz Farhang-Boroujeny ${ }^{\mathrm{a}}$ \\ ${ }^{a}$ Electrical and Computer Engineering Department, University of Utah \\ ${ }^{b}$ Physics and Astronomy Department, University of Utah
}

\begin{abstract}
Cosmic rays are the highest-energy observable particles in the universe. Their study opens a new frontier for scientists to better understand the nature of the universe. This paper reports our study of a bistatic radar approach that is being developed for remote sensing of cosmic-ray induced air showers. In this context, we propose a robust detection technique based on timefrequency domain for the received radar echoes. These echoes are modeled as linear-downward chirp signals, characterized by very short sweep periods, low energies, and corrupted by non-stationary and non-Gaussian background noise. In addition, the related parameters of the received echoes are variable within some expected ranges, determined by the physical parameters of the air showers. In this paper, we explore the performance of the proposed detection method through an extensive theoretical analysis. We derive formulae for probability of the correct detection, as well as false-alarm rate. Numerical simulations and experimental results that corroborate our analysis are also presented.
\end{abstract}

Keywords: Cosmic rays, Chirp detection, Bistatic radar, Hough transform, non-Gaussian noise, low SNR.

\section{Introduction}

Chirp signals are ubiquitous in nature. They can be observed in various areas, such as echolocation (bats) [1], geophysics [2], underwater explorations [3], and gravitational waves in astrophysics [4]. Also, they are frequently encountered in various areas of signal processing, such as sonar 
[5], radar [6], and spread spectrum communications [7, 8]. Some of these applications rely on chirp signal transmission as in the case of sonar [5], while others model the received signal after Doppler spread as chirp signals, e.g., in synthetic aperture radars (SARs) [9], and heart sound signals [10]. In this paper, we study the detection of chirp signals that are encountered in a bistatic radar which we are developing for remote sensing of cosmic ray induced air showers. Next, we discuss the background and motivation behind the presented work in this paper.

\subsection{Background and Motivation}

High energy cosmic rays (HECR) have been a subject to study for many years. Their study is considered to be a major step forward in understanding the fundamental nature of the universe [11]. HECR define high energetic particles, much higher than the energy produced by terrestrial particle accelerators, of extra-terrestrial origin. The origin and composition of the highest energy cosmic rays is currently unknown. When cosmic rays experience collisions with atoms of the upper atmosphere (about $10 \mathrm{~km}$ above the ground), they generate cascades of secondary particles known as extensive air showers (EAS) that propagate through the atmosphere towards the Earth's surface. EAS resulting from cosmic rays produce ionization columns which are detected by such conventional observatories as ground surface-detector arrays and fluorescence detectors. Currently, the Telescope Array (TA) detector, which has been operating in Utah since 2007, employs two detection mechanisms: three fluorescence detectors (FDs) that record the ultraviolet light (UV) emitted from EAS, and a grid of scintillation detectors (SDs) that measures the flux of secondary charged particles arriving at the surface [12]. Fluorescence detection is costly and has a low duty cycle (about 10\%), since the observations can only be made on clear moonless nights. Scintillation detectors operate with 100\% duty cycle, but must cover hundreds or thousands of square kilometers in order to obtain reasonable detection rates. Therefore, a clear motivation exists for moving towards a simpler and more efficient technique such as radar. Our research group, the Telescope Array RAdar (TARA) project, is working on a novel approach based on bistatic radar technology [13]. This technique is promising. It will allow the next generation of cosmic-ray observatories to be built at a fraction of the cost required by current technologies. An additional point to note is that our system setup is co-located with the TA detector, hence allows us to validate the accuracy of the radar system that we are developing. 


\subsection{The Research Problem}

Radar detection of EAS is expected to be a workable solution because of the large ionization densities, at the core of the air shower, which can reflect radio frequencies that lie in the low VHF band. The choice of low $\mathrm{VHF}$ band returns to the corresponding plasma frequency which is of order $50 \mathrm{MHz}$ in which range reflection will occur [14]. In a sufficiently radio-quiet environment, EAS ionization is detectable using radar techniques to capture reflected RF radiation. Research studies have shown that the radar cross section is greatest in the forward scattering direction [15] and hence, bistatic or two-station radar is perfect choice in detecting weak returning echoes. Based on the physical characteristics of cosmic ray air showers, radar echoes are expected to be characterized by a rapid phase modulation-induced frequency shift, covering several tens of $\mathrm{MHz}$ in a period of 10 to $15 \mu \mathrm{sec}$. These signals sweep linearly from a high to low frequency, hence, can be modeled as linear-downward chirps. However, the related parameters of the received echoes are nondeterministic, since they are tied to the physical parameters of the air showers. Thus, unlike most of the existing chirp applications, we are interested in the detection of chirp echoes of variable parameters, center frequencies and frequency rates, within a relatively wide range. In addition, our detection threshold is required to be set as low as possible in order to enhance the ability of detecting signals with signal-to-noise ratio (SNR) in the negative $\mathrm{dB}$ range.

Based on collected data from conventional cosmic-ray observatories, chirpechoes that correspond to high-energy cosmic rays are expected to be rare, at the level of few events per week, and thus, background noise reception is the case most of the time. That makes our radar problem unique and more challenging. Based on our radar environment, background noise is punctuated with persistent single-frequency tones that might originate from different sources around the receiver unit including the radar carrier signal $54.1 \mathrm{MHz}$. These deceptive tones are powerful, hence may lead to positive false-alarms. The other major source of false-alarm is the sudden noise spikes that cover wide frequency bands. These spurious signals cause an erroneous radar detection decision by exceeding the detection threshold. In this research, we address these issues by proposing a robust signal processing technique for detecting the radar echoes and dealing with existing system challenges. 


\subsection{Review of Literature}

In a radar problem, the transmitted signal will be subject to a phase shift induced by the distance and relative motion between the target and the receiver. Thus, a chirp signal may be observed. The phase angle of the chirp reflects the related parameters of the radar target including the speed and range. The estimation of parameters is part of our planned research and thus will be reported in our future publications. In the current phase of our radar application, our main interest lies in the detection of the received chirp echoes produced by cosmic-ray induced air showers.

Different techniques have been developed for the detection of linear chirp signals. The developed methods may target the detection problem in different domains: time-domain or frequency-domain or joint time-frequency domain.

The time domain methods include several adaptive algorithms that approach the detection problem as a recursive least squares (RLS) algorithm [16], or a least mean square (LMS) algorithm [17], and a multiple frequency tracker [18]. However, these adaptive techniques suffer performance degradation under low-SNR conditions [19]. Furthermore, matched filters are one of the most commonly used time-domain methods in radar systems. If the parameters of the radar chirp echoes are known, the optimal detector in stationary white Gaussian noise is proven to be a matched filter followed by a threshold comparison [20]. Passing the chirp echo through its corresponding matched filter will result in a high peak at the output of the filter. This output is known as the chirp autocorrelation function which is well studied in many contexts, e.g., [20]. Neyman-Pearson criterion, or likelihood ratio test, is commonly used in evaluating the detection performance of the matched filter for the case of deterministic chirp signal [21]. In the case of multiple deterministic chirp signals, generalized likelihood ratio test (GLRT) detector is considered where a bank of matched filters are used, [21]. A mismatch of chirp rates between the received signal and the matched filter will result in phase error and thus, a loss in the output peak value. In our application, as mentioned before, we lack the knowledge of received signal parameters and thus, mismatch of the chirp rate between the received chirp signal and the matched filter(s) at the receiver is unavoidable. The assumption of white Gaussian noise is frequently used in the study of radar and communication systems to greatly simplify their analysis. For various applications, the Gaussian noise assumption is justified, e.g., in microwave terrestrial or satellite links; however, in other cases, including ours, background noise turns to be 
impulsive and thus of a non-Gaussian nature. These challenges lead us to consider different approaches.

Discrete Fourier transform (DFT) is considered as a standard, as well as useful, tool for spectrum analysis in the area of digital signal processing, typically implemented in efficient way using Fast Fourier Transform (FFT). Fourier transform provides the corresponding magnitude and phase of the signal spectral content. However, it does not provide a time distribution of the spectral components which we would need for linear chirp signals to look at the change of frequency versus time and exploit the hidden features of chirp signals. In the past, joint time-frequency domain (TFD) techniques have been developed for that purpose. These methods apply a transformation such as the short-time Fourier transform (STFT) or the WignerVille Distribution (WVD) to obtain the time-frequency information. Among these techniques, Page's test [22], the expectation-maximization (EM) algorithm [23], and Hough transform (HT) [24] are more broadly used. Other two-dimensional techniques include Radon transform [25] and Radon-Fourier transform [26]. These approaches are more robust than time-domain methods in detecting non-deterministic chirps and overcoming the limitations of matched filters.

\subsection{Paper Contributions}

In this paper, we focus our attention to the detection of linear chirp signals using a Hough-transform based technique and propose a strategy for detection of chirp signals with non-deterministic parameters (center-frequencies and chirp-rates). We introduce additions to the proposed detector that optimize the detection performance and greatly reduce the computational complexity. Without loss of generality, we consider the bistatic radar application which we are developing for remote sensing of cosmic-ray induced air showers as a good example in evaluating the performance of our proposed technique. For the case where background noise is additive, white, and Gaussian, we develop analytical expressions for the probability of false-alarm and the probability of correct detection of chirp signals. We illustrate the validity of the white Gaussian noise assumption by showing how our proposed technique efficiently filters the existing non-Gaussian components.

In [27], Kay presented GLRT for detection of chirp signals with nondeterministic parameters. In the absence of a uniformly most powerful test (UMPT), GLRT is considered as it tends asymptotically to be optimal [21]. However, it is equivalent to integrating over all possible lines in 
time-frequency domain which is computationally complex and not feasible to implement. In designing our technique, we consider Hough transform capable of two main aspects. First, it uses a time-frequency domain selection criteria that would enable us to integrate over the signal of interest only, which makes our technique feasible to implement as a real-time application. Second, we design the detection method to filter spurious noise components, which reduce the number of received false-alarms and allow us to lower detection threshold.

Different time-frequency representations have been studied in literature [28]. Spectrogram and WVD are widely used due to their simplicity. In our analysis, we use spectrogram to represent the signal in time-frequency plane. Although, WVD is proven to have a higher time-frequency resolution, it suffers from complexity and cross terms [29] which distorts the signal of interest, especially when multiple components exist.

On the other side, Barbarossa in [30] presented Hough transform along with Wigner-Ville distribution for the detection and parameter estimation of unknown signals embedded in noise. Similar work is presented in [31]. However, these works lack theoretical analysis and the study of detection performance. In [32], Hough transform is used in the context of radar for detecting target range signatures, which are characterized by linear slope for stationary and uniform velocity targets. In deriving the probability of falsealarm, we find the described analytical approach in [32] valid for our case due to same white noise assumption. However, for deriving the probability of correct detection, our analytical results are different due to different power distribution of the linear signatures. The developed theoretical results are confirmed through computer simulations. Finally, we introduce the bistaticradar experiment and evaluate the detection performance of the proposed detector under real experimental data and compare it to our previously introduced likelihood ratio test (LRT) detector [33].

\subsection{Organization}

This paper is organized as follows. Section 2 introduces the proposed HT-based detection algorithm. In Section 3, system challenges and design proposals are discussed. In Section 4, the detection performance is analyzed. In Section 5, numerical and simulation results are presented. In Section 6, our actual radar system performance is discussed under real experimental data. Finally, conclusions are drawn in Section 7. 


\subsection{Notations/Terminology}

In our detection problem, two different signal spaces are considered. The first space is obtained by applying DFT to segments of the received signal. This is referred to as TF-space. The second space is obtained by applying Hough-transform to points in TF-space, hence termed as HT-space. We note that here we are dealing with discrete-time signals in both TF-space and HT-space. Accordingly, for each sample sets, TF-space and HT-space are represented by a pair of matrices. For clarity of presentation, we refer to the elements of TF-space matrix as points, and to the elements of HT-space matrix as cells.

In processing TF-space points, we introduce a TF-threshold $\gamma$ and only points which exceed $\gamma$ are Hough-transformed. We also introduce an HTthreshold $\zeta$ and whenever the amplitude of a cell in the HT-space exceeds $\zeta$, signal detection is declared.

In this paper, we evaluate the detection performance of the proposed detector. A false-alarm is declared when in the absence of a chirp signal, one of the cells in the HT-space exceeds $\zeta$, and probability of false-alarm (PFA) is measured accordingly. The average number of false detections per unit of time is defined as the false-alarm rate (FAR). In presence of a linear chirp, when the amplitude of at least one of the cells in HT-space exceeds $\zeta$, we say a correct detection has occurred, and accordingly define the probability of correct detection (PCD). The complement of PCD is termed probability of missed-detection (PMD).

\section{HT-Based Detection Algorithm}

Hough transform (HT) is a pattern detection technique that is commonly used in digital signal and image processing. It is frequently used as a robust method for finding lines in noisy backgrounds [24, 34]. The main premise of the technique is transforming the spatial image pattern into a space of possible parameter values [35]. In that sense, HT converts a complex pattern detection problem in time-frequency space "TF-space" into a simple peak detection problem in Hough-transform space "HT-space". In the following context, we focus, in particular, on using HT for linear chirp detection. TFspace in this case is the time-frequency representation of the received signal samples, and the variables of HT-space are those used to determine the linear variation of the instantaneous frequency in TF-space, viz., chirp parameters. 
The TF-space is represented by $(t, f)$, where $t$ is the time and $f$ is the frequency; see Fig. 1(a). The HT-space, on the other hand, is represented by $(\kappa, c)$, where $\kappa$ is the linear chirp slope and $c$ is the chirp intercept (the intercept of the line with the $f$ axis in the $(t, f)$ plane or the initial frequency.

In our analysis, we use short time Fourier transform (STFT) as a mean of signal evaluation in time-frequency plane. The incoming data samples, symbolized in vector format as $\mathbf{x}$, are divided into overlapping data blocks in time. Each data block "segment" is chosen to be short enough so that the signal can be considered stationary within the segment. The choice of the data block size is based on a tradeoff between temporal and frequency resolution. For each data block, a windowing function $\mathbf{w}$ is applied to localize the signal energy in time and then N-point DFT is applied to each windowed segment.

The N-point discrete-time STFT at time $m$ can be described mathematically as [36]

$$
X_{n, m}=\sum_{i=0}^{N-1} x_{i} w_{i-m} e^{-j 2 \pi n \frac{i}{N}}, \quad n=0,1, \ldots, N-1
$$

where $n$ and $m$ denote the corresponding frequency index and data-block index, respectively. The window sequence $w_{m}$ is assumed to be non-zero in the interval $[0, N-1]$, where $N$ is the number of window samples.

In our application, expected radar echoes lie within a specific frequency band. We take advantage of this feature by only considering the bandwidth of interest. We denote the indices of the start frequency and the end frequency as $n_{1}$ and $n_{2}$, respectively, and we note that $N_{b}=n_{2}-n_{1}+1$ is the number of frequency indices that cover the band of interest.

Complex values $X_{n, m}$ are considered as elements of a matrix $\mathbf{X}$, known as spectrum matrix, that we refer to as

$$
\mathbf{X}=\left[X_{n, m}\right], \quad n=n_{1}, n_{1}+1, \ldots, n_{2} ; m=0,1, \ldots, M-1
$$

where $M$ defines the number of recorded time-blocks. The magnitude squared of the elements of $\mathbf{X}$ yields to the TF-space matrix

$$
\mathbf{S}=\left[\left|X_{n, m}\right|^{2}\right], \quad n=n_{1}, n_{1}+1, \ldots, n_{2} ; m=0,1, \ldots, M-1 .
$$




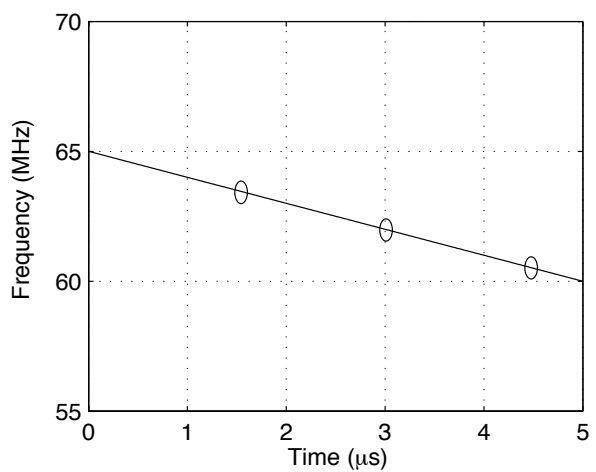

(a)

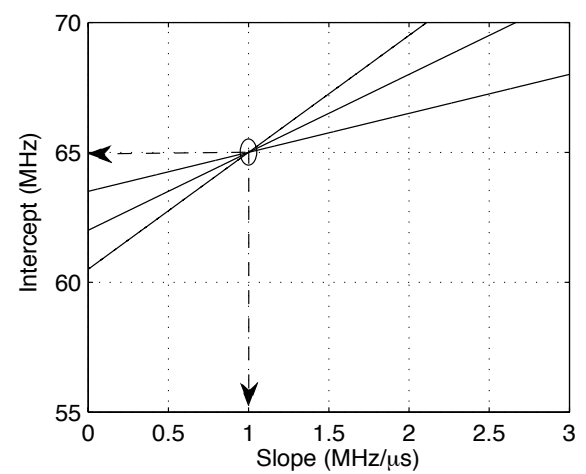

(b)

Figure 1: Illustration of HT for a linear downward chirp with slope $-1 \mathrm{MHz} / \mu s$, start frequency $65 \mathrm{MHz}$, and bandwidth $5 \mathrm{MHz}$.

Each element in $\mathbf{S}$ is compared to the TF-threshold $\gamma$. We choose $\gamma$ as $\gamma_{0}$ times $\eta$, where $\eta$ is the mean of the entries of $\mathbf{S}$. The parameter $\gamma_{0}$, thus, may be thought as the normalized version of $\gamma$ with respect to the background noise. This way, the HT scheme that we adopt is capable of tracking the noise level. Time-frequency points that exceed $\gamma$, signified by the parameters $\left(t_{m}, f_{n}\right)$, known as seed points, are transformed into the following lines in HT-space

$$
c_{k}=f_{n}-t_{m} \kappa_{k} .
$$

Moreover, each line is weighted by the amplitude of its corresponding seed point in the TF-space matrix. The mapped lines to HT-space are then integrated to form the accumulator-matrix, which is initially reset to zero. For more clarity, equation (4) can be manipulated in the following form

$$
f_{i}=\kappa_{k} t_{i}+c_{k}, \quad i=n_{1}, n_{1}+1, \ldots, n_{2}
$$

which shows that a single cell $\left(\kappa_{k}, c_{k}\right)$ in the HT-space corresponds to a single straight line in the TF-space. Accordingly, lines of the HT-space that are mapped from collinear points in the TF-space all intersect at a common cell in the HT-space resulting in a local peak as shown in Fig. 1(b). If the peak is detected, the corresponding line in TF-space can be easily located.

In the case of deterministic chirp detection, the cell in HT-space at which the peak happens is known. However, in our case, we are interested in 


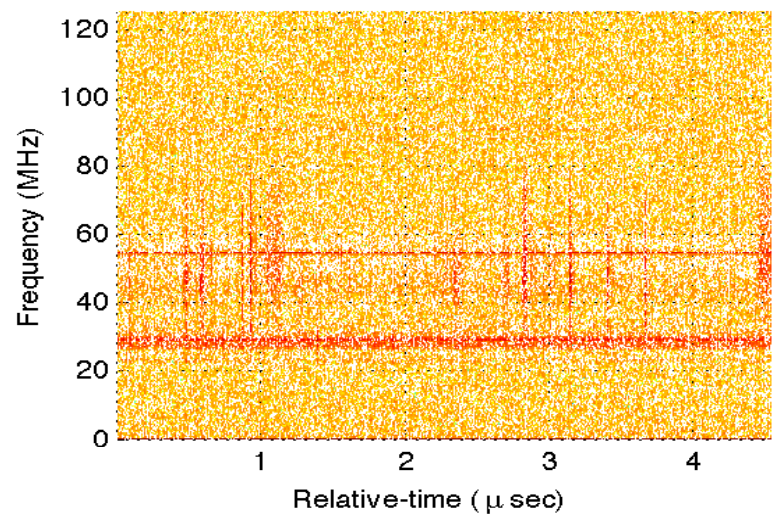

Figure 2: Spectrogram of background noise at the field.

detecting a linear chirp signal with unknown parameters and thus, the local peak detection problem is translated to a peak searching over a range of locations. Detection occurs when the amplitude of any of the accumulatormatrix cells exceeds $\zeta$. We choose $\zeta=\zeta_{0} \gamma$, where $\zeta_{0}$ may be thought as a normalized version of $\zeta$. Also, recalling the relationship $\gamma=\gamma_{0} \eta$, one may note that the choice of $\zeta$ is also relates to the level of the background noise, hence, the method adopted in this paper has a noise tracking capability.

The choice of the pair of thresholds $(\gamma, \zeta)$ is subject to a compromise between aiming at high detection efficiency to avoid missing real radar events and maintaining a low level of false-alarms. In Sections 4 and 5, we discuss in detail how we choose the pair of thresholds.

\section{Radar Background Challenges}

In our application, radar events are very rare, probably a few events per week, and occur at random instants of time. Thus, noise reception is expected to be dominant. This challenge gives rise to the need for smart background analysis to reduce false-alarms while keeping the detection threshold as low as possible to avoid miss detection of the rare events. Fig. 2 shows a sample of an acquired data at the field, where FM radio signal range is filtered out. From the time-frequency representation, we can deduce that the noise background is rich with multiple undesirable components including stationary signals as the persistent frequency tones located at $28.5 \mathrm{MHz}$ and $54.1 \mathrm{MHz}$ as well as 
the broadband transient signals that are randomly located in time domain. In this section, we investigate the background signal components and explore some additions to the HT detector for reducing the number of false-alarms, speeding-up the detection test, yet without degrading the detection efficiency.

\subsection{Powerful Tones}

When frequency-tones are received within the band of interest, they share the linear variation in time-frequency plane with the expected linear-chirps, but with a zero slope. These tones can be easily discerned in the HT-space and their effect can be alleviated by neglecting their corresponding localpeaks located at $(\kappa=0)$ cells so that other peaks can be investigated using the same procedure. However, the strength of powerful tones lies beyond their misleading local-peaks as they also result in a grid of high-variance cells that may accumulate in time and result in false peaks that are randomly located. Thus, we identify the presence of powerful tones for each computed TF-space matrix then the detected components are excluded from the test, simply by forcing them to zero, so that other time-frequency points can be investigated more accurately.

\subsection{Noise Spikes}

Transient signals "spikes" are very common in our scenario and they represent the main source of false-alarms due to their coverage of wide frequency bands. Consequently, the threshold of our proposed detector must be raised in order to keep the desired level of false-alarm rate sufficiently low. This challenge gives rise to the need for smart analysis to reduce false-alarms while keeping the detection threshold as low as possible to avoid miss detection of the rare events. Narrow spikes are also featured by their high slopes in timefrequency plane (almost vertical lines). After Hough-transformation, they produce local-maxima in the accumulator-matrix, which shall be collocated at the maximum slope-points. Consequently, they can be neglected due to the prior knowledge of the expected range of chirp-slopes and then searching process for chirps continues.

\section{Detection Analysis}

In the previous section, we explored the time-frequency domain features that efficiently filter the undesirable noise components, and hence, our radar problem is reduced to signal detection in a white Gaussian noise background. 
First, we note that the signal of interest has the following form in timedomain

$$
c_{i}=\operatorname{rect}\left(\frac{i}{N_{c}}\right) \cos \left(\theta_{i}\right), \quad i=0,1, \ldots, N_{c}-1
$$

where rect( $(\cdot)$ denotes the rectangular function, $i$ denotes the time index, $N_{c}$ denotes the number of chirp signal samples, and the time-varying phase $\theta_{i}$ is expressed as

$$
\theta_{i}=2 \pi f_{\mathrm{C}} i-\pi \kappa i^{2}, \quad i=0,1, \ldots, N_{c}-1
$$

where $f_{\mathrm{C}}$ denotes the chirp center frequency, $f_{\mathrm{H}}$ start (high) frequency and $f_{\mathrm{L}}$ end (low) frequency.

The received signal $x_{i}$, thus, is modeled as

$$
x_{i}=c_{i}+\nu_{i}, \quad i=0,1, \ldots, N_{c}-1
$$

where $\nu_{i}$ is an additive white Gaussian noise (AWGN). Vector $\mathbf{x}$ used in Section II represents the overlapping data blocks of the received signal $x_{i}$. For our radar application, we consider two received signal hypotheses: either noise only $\left(H_{0}\right)$ or signal-plus-noise $\left(H_{1}\right)$. As mentioned in Section 2, a detection is declared when the amplitude of any of the accumulator-matrix cells exceed the HT-threshold.

For $H_{0}$, we wish not to detect anything and thus, minimize false-alarms. However, for $H_{1}$, we wish to detect the chirp signal with a high probability, when it exists. In other words, we wish to choose the pair of thresholds $(\gamma, \zeta)$ such that for a given signal-to-noise ratio (SNR), they lead to a PCD close to one, while keeping a reasonably low value of PFA. The pair of thresholds' selection is subject to a compromise between aiming at high detection efficiency to avoid missing real radar events and maintaining a low level of false-alarms to keep storage low and, hence, accelerating the offline processing of the stored data.

\section{1. $H_{0}$ : chirp signal absence}

We assume the Gaussian noise samples $\nu_{i}$ have a zero mean, are independent and identically distributed, and have a variance of $\sigma_{\nu}^{2}=1$. This leads to Rayleigh-distributed noise spectral amplitudes. Thus, one can deduce that 
the corresponding elements of the TF-space matrix $\mathbf{S}$ are exponentially distributed [[37], p. 78, Eq.(3.33)]. Hence, the probability of each element of $\mathbf{S}$ exceeding the TF-threshold $\gamma$ is obtained as

$$
\begin{aligned}
P_{\mathrm{n}} & =P(s>\gamma) \\
& =\int_{\gamma}^{\infty} e^{-s} d s \\
& =e^{-\gamma}, \quad 0<\gamma<\infty
\end{aligned}
$$

Here, the subscript 'n' is to signify that the probability term arises from noise only. Each time-frequency point exceeding $\gamma$ contributes to a number of cells in the HT-space. We denote the maximum number of time-frequency points that can participate to a given cell in the HT-space as $L_{j}$, where $j$ is the cell index. We note that $L_{j}$ varies from one cell to another.

\subsubsection{Single Cell}

First, we focus our interest on studying a given cell in the HT-space that corresponds to an arbitrary chirp intercept with index $n_{0}$ and chirp rate with index $k_{0}$. In this case, $L_{j}$ equals $L_{0}$. Consider the case where $l$ seed points out of $L_{0}$ to have exceeded the TF-threshold and use $s_{i}$ to denote the corresponding elements of $\mathbf{S}$. The accumulated cell value in the HT-space is then given by

$$
a_{0}=\sum_{i=1}^{l} s_{i}
$$

Next, we note that

$$
P\left(a_{0}>\zeta \mid H_{0}\right)=\sum_{l=1}^{L_{0}} P\left(\forall i: s_{i}>\gamma \mid H_{0}\right) P\left(a_{0}>\zeta \mid\left\{\forall i: s_{i}>\gamma\right\}\right)
$$

where, here, $\forall i$ is the shorthand notation for "for all values of $i$ in the range of 1 to $l$ ". Using (9), the first probability on the right-hand side of (11) is calculated as

$$
P\left(\forall i: s_{i}>\gamma \mid H_{0}\right)=\left(\begin{array}{c}
L_{0} \\
l
\end{array}\right) P_{\mathrm{n}}^{l}\left(1-P_{\mathrm{n}}\right)^{L_{0}-l}
$$


The second probability term on the right-hand side of (11) can be evaluated as

$$
P\left(a_{0}>\zeta \mid\left\{\forall i: s_{i}>\gamma\right\}\right)=\int_{\zeta}^{\infty} P\left(a_{0} \mid\left\{\forall i: s_{i}>\gamma\right\}\right) d a_{0} .
$$

Since $s_{i}$ 's are independent random variables then the probability distribution of their sum, $a_{0}$, is the result of convolving the probability distributions of individual $s_{i}$. The probability density function (PDF) of a single random variable $s_{i}$ given that $s_{i}$ exceeded $\gamma$ is

$$
\begin{aligned}
P\left(s_{i} \mid s_{i}>\gamma\right) & =\frac{P\left(s_{i}\right)}{P\left(s_{i}>\gamma\right)}, \quad s_{i} \in[\gamma, \infty) \\
& =\frac{P\left(s_{i}\right)}{P\left(s_{i}>\gamma\right)} u\left(s_{i}-\gamma\right) \\
& =e^{-\left(s_{i}-\gamma\right)} u\left(s_{i}-\gamma\right)
\end{aligned}
$$

where $u(\cdot)$ denotes the unit step function. We note that the convolution of $l$ exponential variates leads to a chi-squared distribution with $2 l$ degrees of freedom [38]. Hence,

$$
P\left(a_{0} \mid\left\{\forall i: s_{i}>\gamma\right\}\right)=\frac{\left(a_{0}-l \gamma\right)^{l-1}}{(l-1) !} e^{-\left(a_{0}-l \gamma\right)} u\left(a_{0}-l \gamma\right)
$$

Using the PDF in (15), one will obtain the following result

$$
\begin{aligned}
& P\left(a_{0}>\zeta \mid\left\{\forall i: s_{i}>\gamma\right\}\right)=\int_{\zeta}^{\infty} \frac{\left(a_{0}-l \gamma\right)^{l-1}}{(l-1) !} e^{-\left(a_{0}-l \gamma\right)} u\left(a_{0}-l \gamma\right) d a_{0} \\
& =\int_{\max (\zeta, l \gamma)}^{\infty} \frac{\left(a_{0}-l \gamma\right)^{l-1}}{(l-1) !} e^{-\left(a_{0}-l \gamma\right)} d a_{0} \\
& =\int_{\max ((\zeta-l \gamma), 0)}^{\infty} \frac{z^{(l-1)}}{(l-1) !} d z \\
& = \begin{cases}1, & \zeta<l \gamma \\
\frac{\Gamma(l, \zeta-l \gamma)}{(l-1) !}, & \zeta>l \gamma\end{cases} \\
& =u(l \gamma-\zeta)+\frac{\Gamma(l, \zeta-l \gamma)}{(l-1) !} u(\zeta-l \gamma) \text {. }
\end{aligned}
$$


Substituting (12) and (16) in (11), we get

$$
\begin{array}{r}
P\left(a_{0}>\zeta \mid H_{0}\right)=\sum_{l=1}^{L_{0}}\left(\begin{array}{c}
L_{0} \\
l
\end{array}\right) \\
P_{\mathrm{n}}^{l}\left(1-P_{\mathrm{n}}\right)^{L_{0}-l}(u(l \gamma-\zeta) \\
\left.+\frac{\Gamma(l, \zeta-l \gamma)}{(l-1) !} u(\zeta-l \gamma)\right)
\end{array}
$$

where $\Gamma(x, y)$ is the incomplete gamma function, defined as, [39], pp. 899, Eq. (8.352)

$$
\Gamma(x, y)=(x-1) ! e^{-y} \sum_{k=0}^{x-1} \frac{y^{k}}{k !}
$$

\subsubsection{HT-space Matrix}

In the actual problem, a number of cells out of the whole accumulator-matrix are compared against the HT-threshold $\zeta$. A false-alarm is declared if the amplitude of any of these cells, $a_{j}$, exceeds $\zeta$. We denote the number of cells of interest as $N_{s}$, and assume that they are indexed from 1 to $N_{s}$. This leads to the following equation for the probability of false-alarm:

$$
\mathrm{PFA}=1-\prod_{j=1}^{N_{s}} P\left(a_{j}<\zeta \mid H_{0}\right)
$$

where

$$
P\left(a_{j}<\zeta \mid H_{0}\right)=1-P\left(a_{j}>\zeta \mid H_{0}\right)
$$

Next, we need to evaluate $L_{j}$ for each cell $j$ in the accumulator-matrix to be able to obtain $P\left(a_{j}<\zeta \mid H_{0}\right)$ for each. In order to do so, we follow a numerical method similar to the one introduced in [32].

Without loss of generality, we consider our radar application as an appropriate example to understand and visualize the variation of $L_{j}$ along the accumulator-matrix. For the echoes being reflected from cosmic ray induced air showers, the chirp rates of interest belong to the interval $\mathcal{K}=$ $[-3,0] \mathrm{MHz} / \mu \mathrm{s}$. Also, the expected chirp intercept lies in the interval $[55,70] \mathrm{MHz}$. In applying STFT, we consider 256-point DFT size with rectangular windowing and no overlapping between windows. Fig. 3 depicts the corresponding 


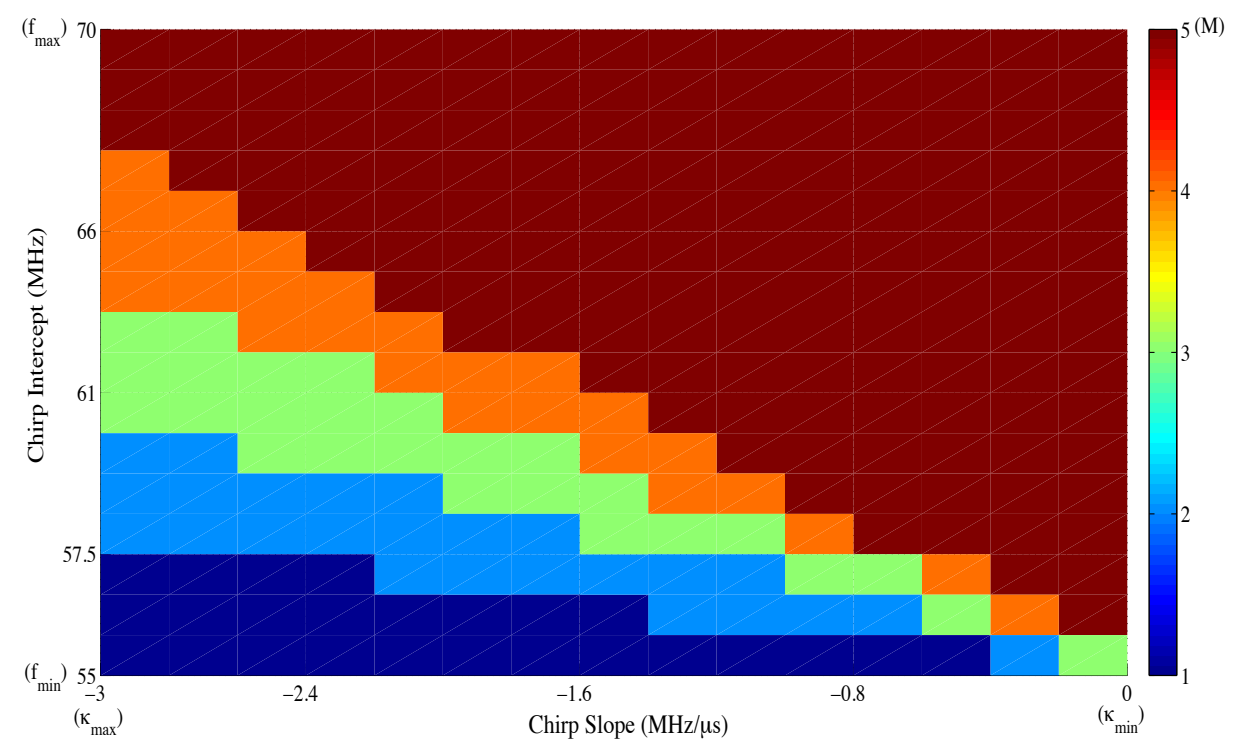

Figure 3: Accumulator-matrix for all points transformed from TF-space. Chirp intercept lies in the interval $[55,70] \mathrm{MHz}$ and chirp rates of interest belong to the interval $\mathcal{K}=[-3,0] \mathrm{MHz} / \mu \mathrm{s} .256$-point DFT is used in generating the STFT and no overlapping between windows.

accumulator-matrix for the case of 5 non-overlapping data blocks $(M=5)$ and $N_{b}=17$. As observed, the contributions received by accumulator-matrix cells vary as the chirp slope varies, as well as the chirp intercept. We can see that accumulator-matrix cells receive less contributions as the chirp slope increases or the chirp intercept decreases, where for both cases; less collinear points constitute the linear chirp in the TF-space. As observed, this contribution varies from 1 to $M$. The cells that receive minimum contribution, $a_{j}=1$, correspond to lines in TF-space that cannot exist so contribution results from one point. However, cells that receive maximum contribution $a_{j}=5$, correspond to lines with maximum number of collinear points in the TF-space which is the number of time-blocks $(M)$ per single TF-space matrix.

For our detection system, as well as in other real-time applications, system user is more interested in evaluating the false-alarm rate (FAR) instead of the probability of false-alarm. FAR defines the average number of false detections, under chirp signal absence, per unit of time. Since we assume 
non-overlapping TF-space matrices, FAR can be directly evaluated through the knowledge of probability of false-alarm simply by multiplying PFA and the number of processed TF-space matrices per second.

\section{2. $H_{1}$ : chirp signal presence}

In our analysis, we consider a particular chirp signal with known start and end frequencies $f_{\mathrm{H}}$ and $f_{\mathrm{L}}$, respectively, and known chirp slope $\kappa_{c}$. We assume the time length of the TF-space matrix to be approximately the same as the chirp duration. Also, for a given range of the frequencies $f_{\mathrm{L}}$ to $f_{\mathrm{H}}$, and the specified DFT length, the corresponding frequency indices be $k_{1}$ to $k_{2}$.

First, we wish to study the PDF of the amplitude peaks in the TF-space. Using a rectangular window, the squared spectrum amplitude for the $m^{\text {th }}$ data block is

$$
\left|X_{n}\right|^{2}=\left|\sum_{i=0}^{N-1} x_{i, m} e^{-j 2 \pi n \frac{i}{N}}\right|^{2}, \quad n=k_{1}, k_{1}+1, \ldots, k_{2}
$$

where $x_{i, m}$ contains the chirp signal $c_{i, m}$ and the additive noise $\nu_{i, m}$. Let $C_{n}$ denotes the DFT output due to $c_{i, m}$ and $\nu_{n}$ denotes the DFT output due to $\nu_{i, m}$, hence, $X_{n}=C_{n}+\nu_{n}$. To arrive at a simple expression that facilitates the subsequent analysis of this section, we assume signal and noise in TF-space are independent random variables. Thus,

$$
\begin{aligned}
\mathbb{E}\left\{\left|X_{n}\right|^{2}\right\} & =\mathbb{E}\left\{\left|C_{n}+\nu_{n}\right|^{2}\right\} \\
& =\left|C_{n}\right|^{2}+\mathbb{E}\left\{\left|\nu_{n}\right|^{2}\right\}
\end{aligned}
$$

where $\mathbb{E}\{\cdot\}$ denotes expectation, and replace the terms with the expectation sign on them by their instantaneous values. This leads to the approximation

$$
\left|X_{n}\right|^{2} \approx\left|C_{n}\right|^{2}+\left|\nu_{n}\right|^{2}, \quad n=k_{1}, k_{1}+1, \ldots, k_{2} .
$$

We note that $\left|C_{n}\right|^{2}$ is determined by the known chirp signal. For a chirp signal, this is approximately a constant over the duration of the chirp, [9]. We denote this constant by $d$. We also note that $\left|\nu_{n}\right|^{2}$ is a random variable with an exponential distribution, as discussed in Section 4.1.

Now we need to calculate the probability of a single-point's amplitude exceeding the TF-threshold, $\gamma$. For any given point of TF-space that is 
impacted by both noise and a chirp signal, one finds that

$$
\begin{aligned}
P_{\mathrm{d}} & =P(s>\gamma) \\
& =P((\nu+d)>\gamma) \\
& =\left\{\begin{array}{l}
\int_{\gamma-d}^{\infty} e^{-\nu} d \nu, \gamma>d \\
1, \text { otherwise. }
\end{array}\right. \\
& =\left\{\begin{array}{l}
e^{-(\gamma-d)}, \gamma>d \\
1, \text { otherwise. }
\end{array}\right.
\end{aligned}
$$

In the TF-space matrix, of the $M$ points that are affected by the chirp, $l$ of them may exceed $\gamma$, in amplitude, and thus each is transformed to a line in the HT-space and, accordingly, accumulated. The location of the peak in the HT-space is determined through the knowledge of the chirp intercept $f_{\mathrm{H}}$ and rate $\kappa_{c}$. The probability of the corresponding accumulator-matrix cell $a_{c}$ exceeding $\zeta$ defines PCD and is evaluated as

$$
\begin{aligned}
\mathrm{PCD} & =P\left(a_{c}>\zeta\right) \\
& =\sum_{l=1}^{M} P\left(\forall i: s_{i}>\gamma \mid H_{1}\right) P\left(a_{c}>\zeta \mid\left\{\forall i: s_{i}>\gamma\right\}\right)
\end{aligned}
$$

Similar to (12), the first probability term can be written as

$$
P\left(\forall i: s_{i}>\gamma \mid H_{1}\right)=\left(\begin{array}{c}
M \\
l
\end{array}\right) P_{\mathrm{d}}^{l}\left(1-P_{\mathrm{d}}\right)^{M-l} .
$$

And the second probability term can be evaluated as

$$
P\left(a_{c}>\zeta \mid\left\{\forall i: s_{i}>\gamma\right\}\right)=\int_{\zeta}^{\infty} P\left(a_{c} \mid\left\{\forall i: s_{i}>\gamma\right\}\right) .
$$

As in (14), the PDF of a single random variable $s_{i}$ given that it has been affected by a chirp signal and exceeds $\gamma$ may be written as

$$
\begin{aligned}
P\left(s_{i} \mid s_{i}>\gamma\right) & =\frac{P\left(s_{i}\right)}{P\left(s_{i}>\gamma\right)}, \quad s_{i} \in[\gamma, \infty) \\
& = \begin{cases}e^{-\left(s_{i}-\gamma\right)} u\left(s_{i}-\gamma\right), & \gamma>d \\
e^{-\left(s_{i}-d\right)} u\left(s_{i}-\gamma\right), & \text { otherwise. }\end{cases}
\end{aligned}
$$


For $a_{c}$, similar to the noise analysis, we evaluate the convolution of $l$ exponential variates which leads to a chi-squared distribution with $2 l$ degrees of freedom.

$$
P\left(a_{c} \mid\left\{\forall i: s_{i}>\gamma\right\}\right)= \begin{cases}\frac{\left(a_{c}-l \gamma\right)^{l-1}}{(l-1) !} e^{-\left(a_{c}-l \gamma\right)} u\left(a_{c}-l \gamma\right), & \gamma>d \\ \frac{\left(a_{c}-l d\right)^{l-1}}{(l-1) !} e^{-\left(a_{c}-l d\right)} u\left(a_{c}-l \gamma\right), & \text { otherwise. }\end{cases}
$$

Using the calculated PDF, the second probability term can be also computed as

$$
\begin{aligned}
& P\left(a_{c}>\zeta \mid\left\{\forall i: s_{i}>\gamma\right\}\right)=\int_{\zeta}^{\infty} \begin{cases}\frac{\left(a_{c}-l \gamma\right)^{l-1}}{(l-1) !} e^{-\left(a_{c}-l \gamma\right)} u\left(a_{c}-l \gamma\right) d a_{c}, & \gamma>d \\
\frac{\left(a_{c}-l d\right)^{l-1}}{(l-1) !} e^{-\left(a_{c}-l d\right)} u\left(a_{c}-l \gamma\right) d a_{c}, & \text { otherwise. }\end{cases} \\
& =\int_{\max (\zeta, l \gamma)}^{\infty} \begin{cases}\frac{\left(a_{c}-l \gamma\right)^{l-1}}{(l-1) !} e^{-\left(a_{c}-l \gamma\right)} d a_{c}, & \gamma>d \\
\frac{\left(a_{c}-l d\right)^{l-1}}{(l-1) !} e^{-\left(a_{c}-l d\right)} d a_{c}, & \text { otherwise. }\end{cases} \\
& =\left\{\begin{array}{l}
\int_{\max (\zeta-l \gamma, 0)}^{\infty} \frac{z^{l-1}}{(l-1) !} e^{-z} d z, \quad \gamma>d \\
\int_{\max (\zeta-l d, 0)}^{\infty} \frac{z^{l-1}}{(l-1) !} e^{-z} d z, \text { otherwise. }
\end{array}\right. \\
& =u(\gamma-d)\left(u(l \gamma-\zeta)+u(\zeta-l \gamma) \frac{\Gamma(l, \zeta-l \gamma)}{(l-1) !}\right)+ \\
& u(d-\gamma)\left(u(l d-\zeta)+u(\zeta-l d) \frac{\Gamma(l, \zeta-l d)}{(l-1) !}\right)
\end{aligned}
$$

Substituting (26) and (30) in (25), we get

$$
\begin{array}{r}
\mathrm{PCD}=\left(\begin{array}{c}
M \\
l
\end{array}\right) P_{\mathrm{d}}^{l}\left(1-P_{\mathrm{d}}\right)^{M-l}\left[u(\gamma-d)\left(u(l \gamma-\zeta)+u(\zeta-l \gamma) \frac{\Gamma(l, \zeta-l \gamma)}{(l-1) !}\right)+\right. \\
\left.u(d-\gamma)\left(u(l d-\zeta)+u(\zeta-l d) \frac{\Gamma(l, \zeta-l d)}{(l-1) !}\right)\right]
\end{array}
$$




\section{Numerical Analysis}

Our objective in this section is to demonstrate the detection performance of the proposed detector. In addition, we present numerical results that confirm the validity of our formulated analytical expressions by comparing them with simulation results. In our study, two separate tests are performed. First, we evaluate the performance of the proposed detector under white Gaussian noise. The goal from this test is to measure the probability of falsealarm acquired by the HT-based detector. Second, we measure the detection performance of the detector for different expected linear chirp signals versus SNR under a certain probability of false-alarm.

Before we proceed with the presentation of our numerical results, we make the following observations. It is clear from (19) and (31) that the detection performance of the proposed detector is a function of the pair of thresholds $(\gamma, \zeta)$. In designing our detector, we follow the constant false-alarm rate (CFAR) criterion by using adaptive pair of thresholds $(\gamma, \zeta)$ that keep track of noise variations. Accordingly, $(\gamma, \zeta)$, in (19) and (31) expressions, can be replaced by their normalized pair $\left(\gamma_{0}, \zeta_{0}\right)$. Basically, a detection is declared, either due to noise-only or signal-plus-noise, whenever the accumulation sum of an HT-space's cell exceeds the defined HT-threshold $\zeta$, while at least one TF-space cell has to have exceeded the defined TF-threshold $\gamma$.

System parameters are chosen as follows. The sample rate is 250 mega samples per second (MSPS). The choice of window length of the spectrogram is crucial as it provides a compromise between temporal and frequency resolution: a shorter window size means more temporal localization but less spectral discrimination. For a linear chirp signal of constant amplitude, using a rectangular window, the optimal window length is given by [40]

$$
T_{w}=\sqrt{\frac{2}{|\kappa|}} \quad \text { seconds. }
$$

In order to relate the mentioned formula to our application, we define the expected range of chirp slopes through the physical simulation of our radar target. We focus our interest on the most probabilistic interval $[-3,-1] \mathrm{MHz} / \mu s$. Hence, replacing $\kappa$ by its average value in (32). This leads to $T_{w}=10^{-6}$. Given the sampling rate of $250 \mathrm{MSPS}$, this corresponds to 250 samples, which we round to 256 (the nearest power of 2). We consider the full frequency range for the intercept $[0,125] \mathrm{MHz}$ and $[-3,-1] \mathrm{MHz} / \mu$ s for the chirp slope 


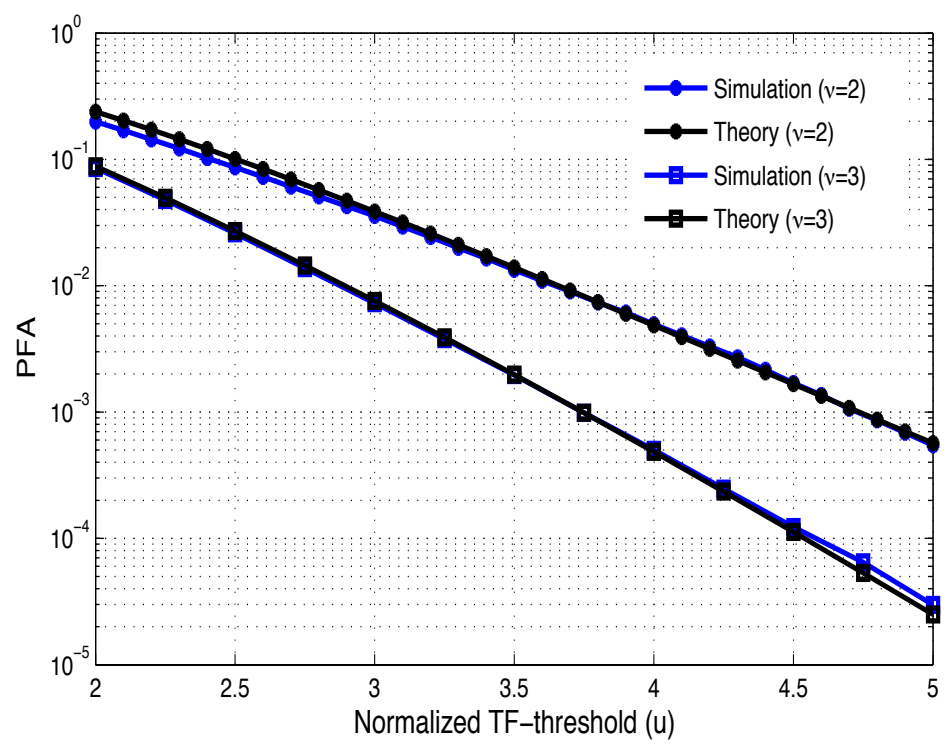

Figure 4: Probability of False-Alarm for a single-cell in the accumulator-matrix versus a range of TF-threshold values under two different HT-threshold values. Simulated and analytical results are plotted over white Gaussian noise.

with a step size of $0.2 \mathrm{MHz} / \mu s$. In addition, we consider signals of interest with fixed bandwidth $5 \mathrm{MHz}$, centered at $62.5 \mathrm{MHz}$. We present results of PFA and PCD for different SNR values in the range of $[-25,10] \mathrm{dB}$.

\subsection{Probability of False-Alarm (PFA)}

Equation (19) provides an expression for the overall probability of falsealarm. As seen, it is a function of the maximum number of time-frequency points, $L_{j}$, TF-threshold $\gamma=u \eta$, and HT-threshold $\zeta=\nu \gamma$. Since $L_{j}$ varies from one cell to another, probability of false-alarm per cell is cell dependent.

We plot probability of false-alarm for a single-cell case, which can be directly extended to the cell-matrix case or the whole accumulator-matrix. Fig. 4 shows the simulated and analytical result of PFA for a single-cell that correspond to chirp intercept $65 \mathrm{MHz}$ and chirp slope $-1 \mathrm{MHz} / \mu \mathrm{s}$. We see that the simulation results match the theoretical results perfectly for the single-cell case.

\subsection{Probability of Correct Detection (PCD)}

We start by looking at the detection efficiency of chirp signals with different chirp slopes. Fig. 5 presents the probability of detection versus SNR, 
for two distinct chirp slopes; $-1 \mathrm{MHz} / \mu s$ and $-1.6 \mathrm{MHz} / \mu s$. Simulation results are plotted along with theoretical results based on the expression given in (31). These results clearly prove the validity of our theoretical calculations. Furthermore, we observe that PCD decreases as the chirp slope increases. This observation can be understood by noting that the higher the chirp slope is, the less will be the seed points that contribute to formation of the local peak in the accumulator-matrix. Next, we perform two

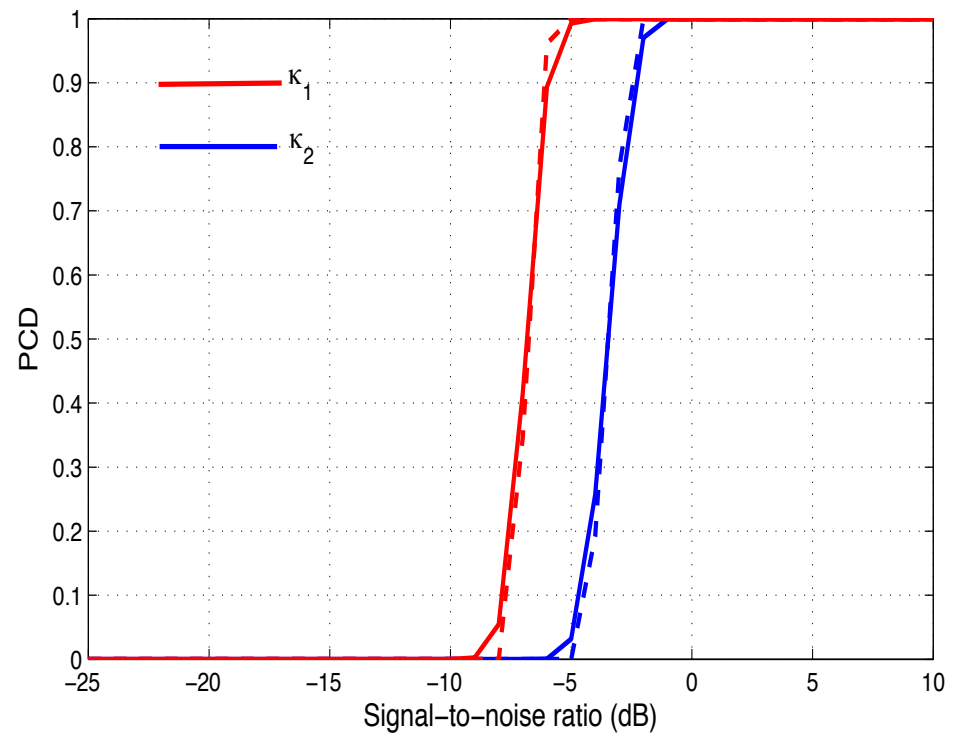

Figure 5: Probability of correct detection versus SNR for two distinct chirp slopes, where $\left(\kappa_{1}, \kappa_{2}\right)$ equals $(-1,-1.6) \mathrm{MHz} / \mu s$, respectively, under $\gamma_{0}$ equals 12 and $\zeta_{0}$ equals 10. Simulated (solid-line) and analytical (dotted-line) results are plotted over AWGN.

separate tests to understand the effect of varying the TF-threshold and the HT-threshold on the detection performance. First, we evaluate the detection performance of a linear chirp signal with a fixed slope $(-1.6 \mathrm{MHz} / \mu \mathrm{s})$ under different TF-threshold levels and a fixed HT-threshold level. The goal here is to estimate the expected deterioration in detection performance by raising the TF-threshold value. Fig. 6 presents PCD plots as a function of SNR for TF-threshold levels $(7 \eta, 10 \eta, 13 \eta)$ and fixed HT-threshold level $(10 \gamma)$. Again, these results show that theoretical calculations can perfectly track simulation results. As observed, the curves shift to right $2 \mathrm{~dB}$ for each $3 \eta$ units increase in the TF-threshold.

On the other hand, we evaluate the detection performance of a linear chirp 


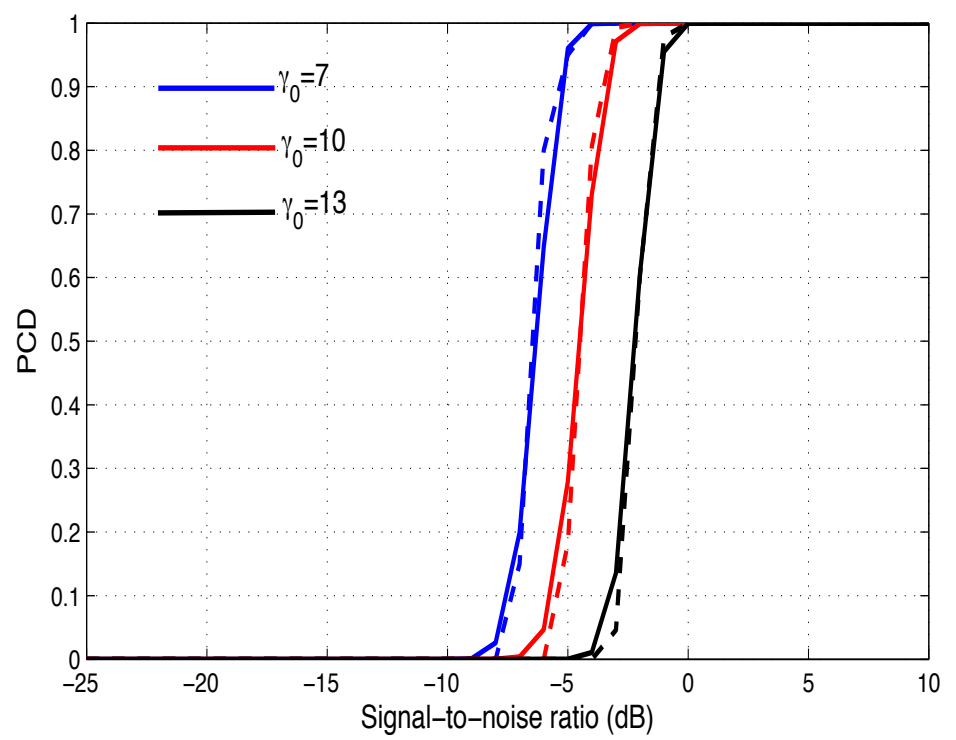

Figure 6: Probability of correct detection versus SNR for three different TF-threshold levels $(7 \eta, 10 \eta, 13 \eta)$ and fixed HT-threshold level $(10 \gamma)$. Simulated (solid-line) and analytical (dotted-line) results are plotted over AWGN.

signal with a fixed slope $(-1.6 \mathrm{MHz} / \mu s)$ under different HT-threshold levels and a fixed TF-threshold level. Fig. 7 presents PCD plots as a function of SNR for HT-threshold levels $(7 \gamma, 10 \gamma, 13 \gamma)$ and fixed TF-threshold level $(10 \eta)$. As observed, the curves shift to right only $1 \mathrm{~dB}$ for each $3 \gamma$ units increase in the HT-threshold. These results show that the TF-threshold has a larger effect on PCD than the HT-threshold.

\subsection{Receiver Operating Characteristic}

Fig. 8 shows the receiver operating characteristic (ROC) curves for the proposed detector. This set of curves show the calculated PCD of a linear chirp signal, with the rate of $-1.6 \mathrm{MHz} / \mu s$, versus the FAR of the HT-based detector for a number of low received SNR values $(-6 \mathrm{~dB},-7 \mathrm{~dB},-8 \mathrm{~dB})$. Each point on each of the ROC curves corresponds to a specific pair of TFthreshold and HT-threshold values. In the plotted curves, the HT-threshold is fixed to $5 \gamma$ and the TF-threshold is varied within $[3 \eta, 15 \eta]$.

As depicted in the figure, we can find a range of TF-threshold values $[4.5 \eta, 6.5 \eta]$ where FAR is in order $10^{-3}$, few events per hour or less, and at the same time, we can achieve complete probability of detection for the transmitted chirp at SNR of $-7 \mathrm{~dB}$. 


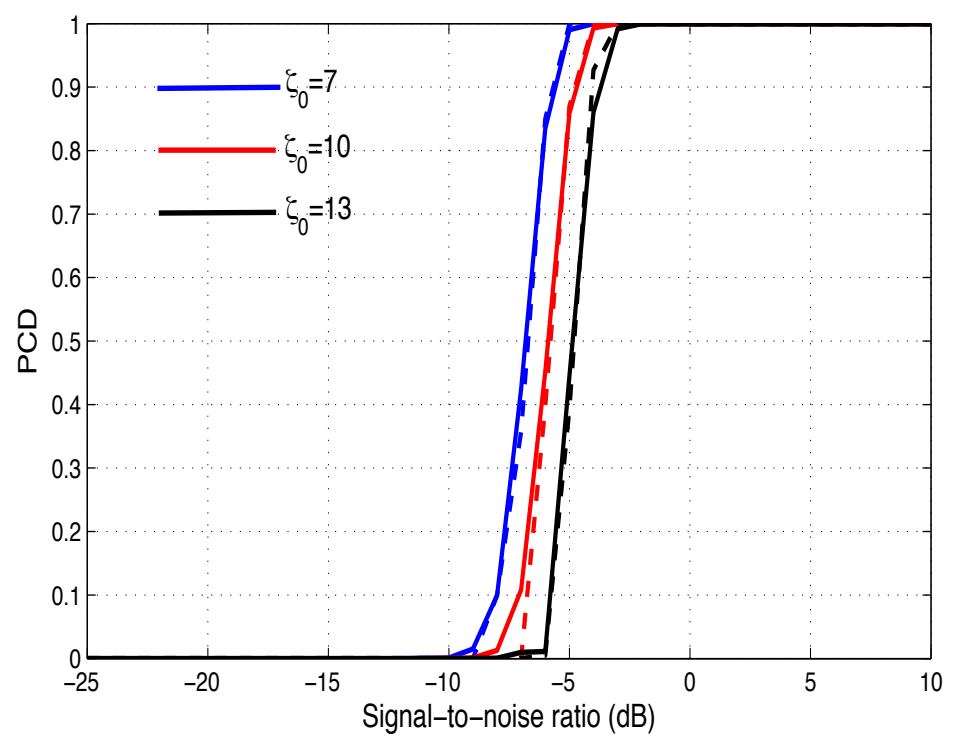

Figure 7: Probability of correct detection versus SNR for three different HT-threshold levels $(7 \gamma, 10 \gamma, 13 \gamma)$ and fixed TF-threshold level 10 $\eta$. Simulated (solid-line) and analytical (dotted-line) results are plotted over AWGN.

\section{Radar System Performance}

Fig. 9 shows the basic elements of our bistatic radar system, located in Delta county, Utah. In building the transmitter station, we make use of analog television transmitters donated to University of Utah by Salt Lake City's KUTV Channel 2 and ABC4 [13]. The transmitter station, operating under FCC license, broadcasts a continuous 54.1 MHz carrier signal with a $40 \mathrm{~kW}$ of power above the Telescope Array surface detectors. Also, our radar receiver station is placed at Long Ridge, $40 \mathrm{~km}$ distant from the transmitter site. We utilize the NI-5761 adapter module with a sample rate $\left(F_{s}\right) 250$ Million samples per second (MSPS). Our system-on-chip design is implemented over the high performance Virtex-5 FPGA which is integrated with the fast PXIe interface for host connectivity. In our study, we consider experimental data acquired at the field using our bistatic radar receiver. We compare the performance of the proposed detector to our previously introduced LRT detector in [33]. For the LRT detector, we use an amplitude limiter to get rid of the impulsive noise. The amplitude limiter clips the amplitude of the received signal to a factor of $k$ of its root mean square (RMS) value before clipping. This would allow us to bring the detection threshold of the LRT 


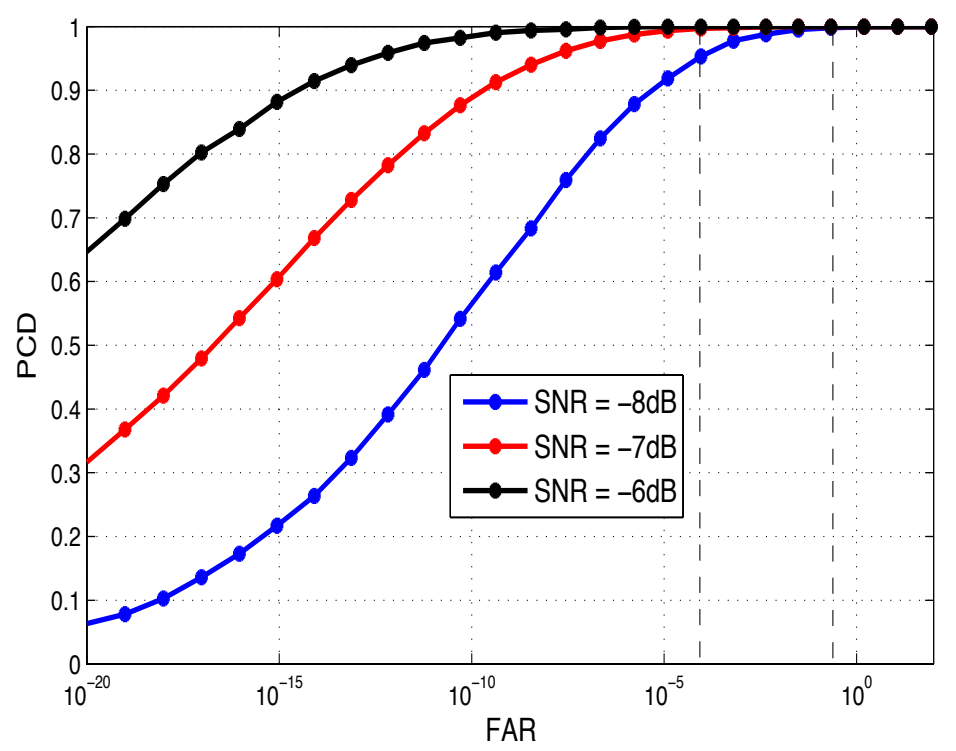

Figure 8: Empirical receiver operating characteristic (ROC) curves. Probability of correct detection is plotted versus false-alarm rate for different number of signal-to-noise ratio (SNR) levels.

detector lower and thus, challenge the detection performance of the proposed HT-based detector.

For radar system testing, we conduct two basic performance tests through a series of radar measurements. First, we evaluate the performance of the proposed detector under the existing non-Gaussian environment. The goal from this test is to measure the probability of false-alarm (PFA) acquired by the HT-based detector. Second, we assess the detection performance of the proposed detector for a typical chirp signal versus SNR under a specified pair of thresholds $(\gamma, \zeta)$ that corresponds to a reasonable level of PFA.

As we noted before, our radar system receives multiple undesirable frequency tones which might originate from different sources around the receiver unit including the powerful radar carrier signal (54.1 MHz). Using the smart features of our detector, these persistent tones can be filtered out. In addition, since we know the band of interest, we apply a digital band-pass filter (60-65) $\mathrm{MHz}$ at the input stage of our detector for noise reduction and carrier suppression.

Fig. 10 depicts the probability of false-alarm versus the TF-threshold $\gamma$ for various HT-threshold values. In our second test, and due to rarity 


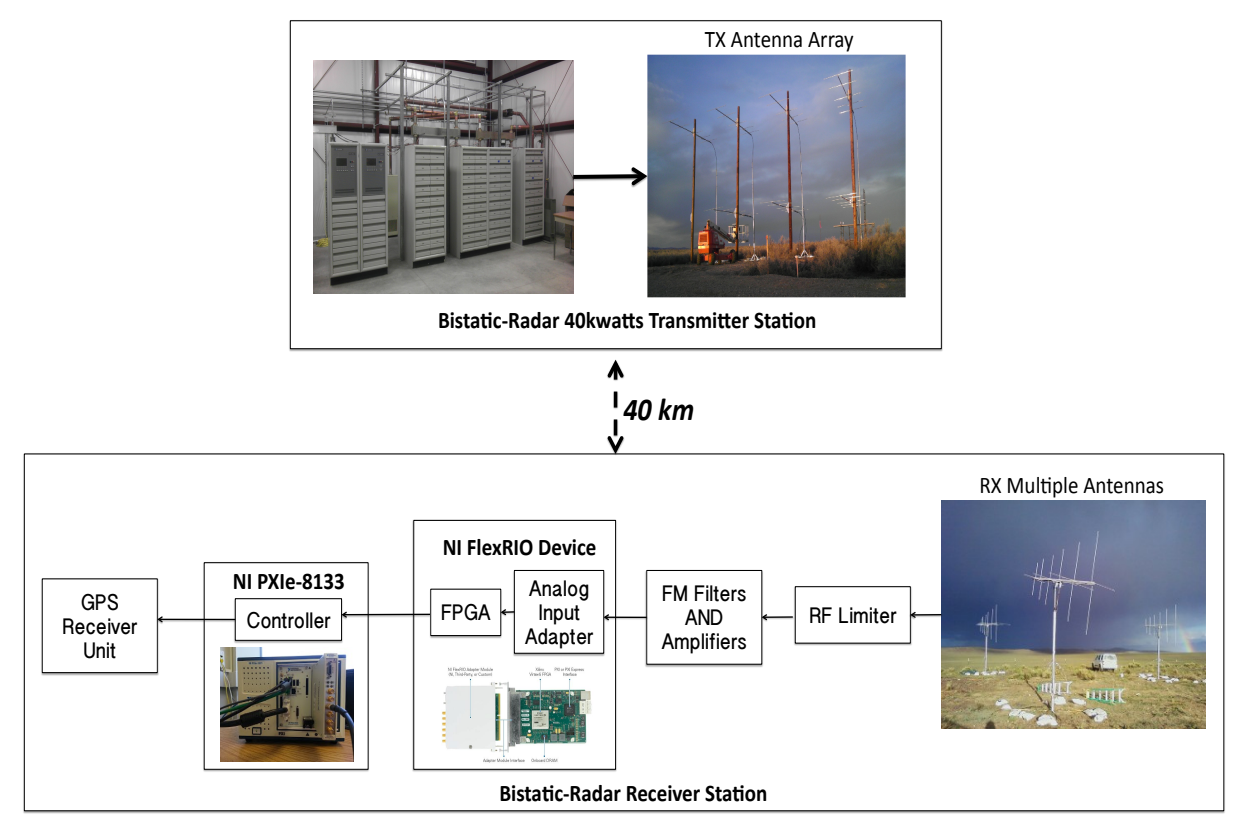

Figure 9: Elements of the bistatic radar system.

of radar echoes, we alternatively placed artificially-generated chirp signals in the same background for evaluating the detection performance. Fig. 11 depicts sample of the acquired data after embedding a linear chirp signal of a typical slope $(-1 \mathrm{MHz} / \mu \mathrm{s})$ and $-10 \mathrm{~dB}$ SNR value. For a fair comparison, we compare the detection performance of both detectors under PFA equals $10^{-3}$ and for a fixed bandwidth $(5 \mathrm{MHz})$ chirps with center frequency $f_{\mathrm{C}}$ equals $62.5 \mathrm{MHz}$. Fig. 12 shows performance comparison of both detectors. As depicted in Fig. 12, the minimum SNR for which complete detection is achieved, is $-4 \mathrm{~dB}$ for the HT-based detector and $8 \mathrm{~dB}$ for the LRT detector which implies that HT significantly enhances detection by a factor of $14 \mathrm{~dB}$. Using the amplitude limiter, the detection performance of the LRT detector is enhanced to a great extent, yet the HT-based detector outperforms by $2 \mathrm{~dB}$.

\section{Conclusion}

The main premise of this paper was to design a smart time-frequency based detection technique for radar echoes of a bistatic radar application which we are developing for remote sensing of cosmic-ray induced air show- 


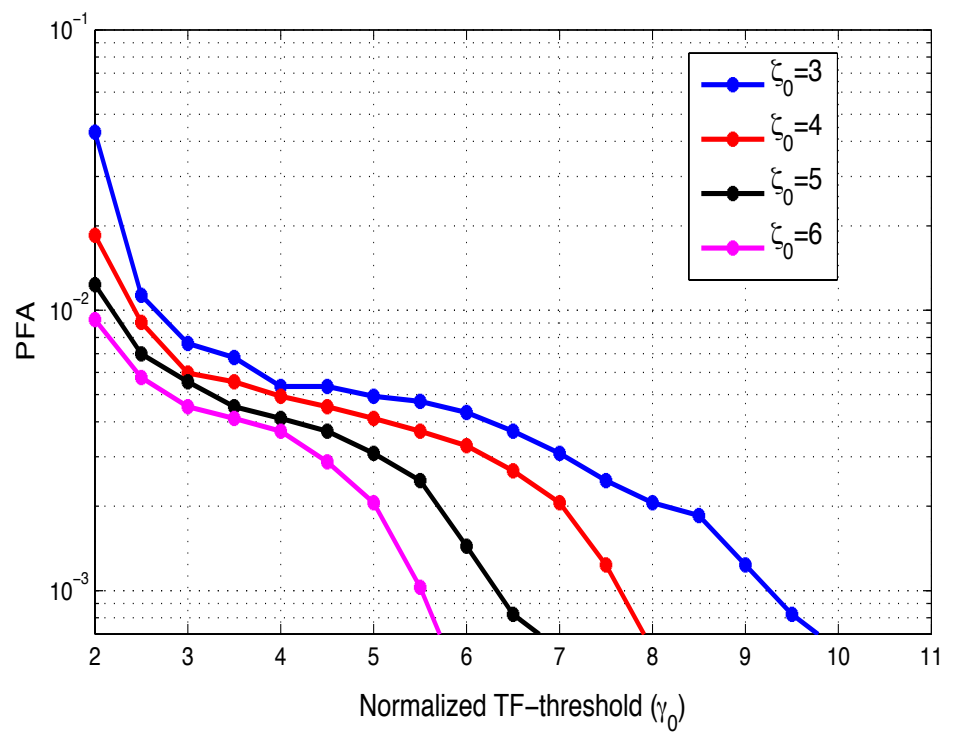

Figure 10: HT detector: probability of false-alarm versus relative TF-threshold ( $\gamma_{0}$ units of the averaged standard deviation of the entries in the TF-space matrix).
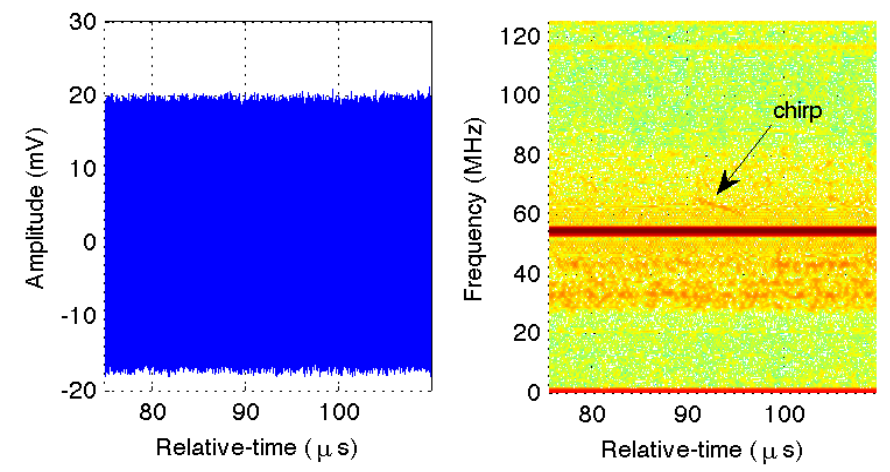

Figure 11: Linear chirp signal added to background noise.

ers. Returning radar echoes are expected to sweep from a high to a low frequency with non-deterministic parameters but known intervals. We used Hough transform as an efficient pattern recognition tool to design our proposed detector. In this paper, we developed a mathematical framework for the design and analysis of the proposed detector. We compared the detection performance of the proposed detector to our previously introduced LRT 


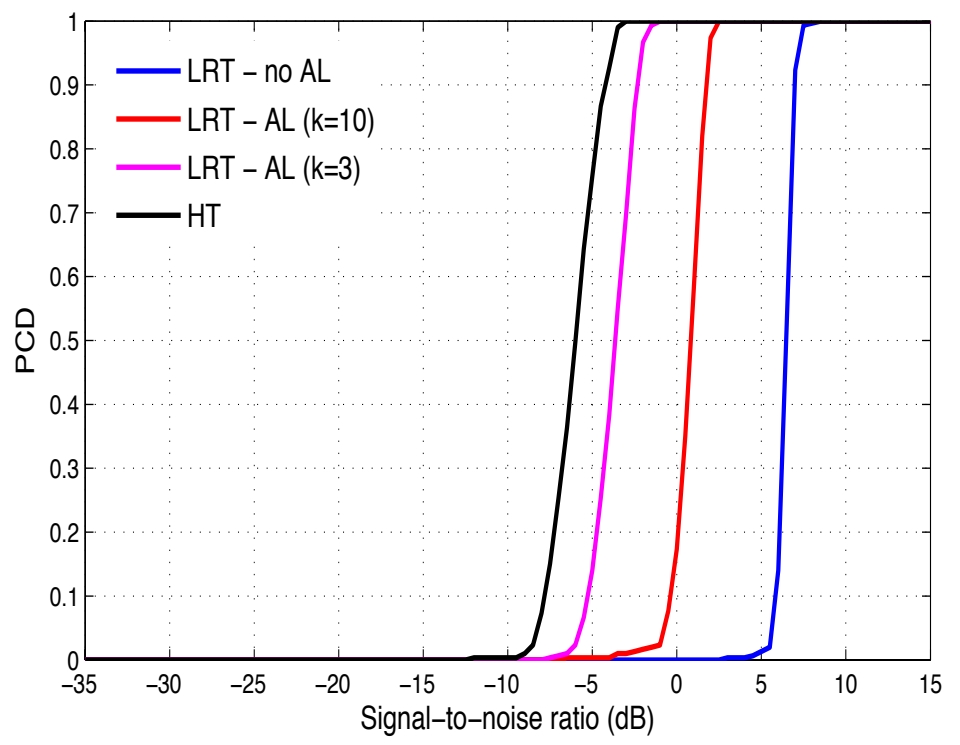

Figure 12: Probability of correct detection for HT-based detector and LRT detector with and without amplitude limiter under PFA equals $10^{-3}$.

detector and we found that the HT-based detector outperforms under the non-Gaussian radar environment. The accuracy of our analytic results were confirmed by running simulations and field experiments.

\section{Acknowledgment}

This work is supported by the U.S. National Science Foundation grants NSF/PHY-0969865 and NSF/MRI-1126353, and by the W.M. Keck Foundation. We also acknowledge valuable discussions with other members of the TARA collaboration. 
[1] R. Altes, Echolocation as seen from the viewpoint of radar/sonar theory, in: Localization and Orientation in Biology and Engineering, Proceedings in Life Sciences, Springer Berlin Heidelberg, 1984, pp. 234-244.

[2] B. Askeland, H. Hobaek, R. Mjelde, Semiperiodic chirp sequences reduce autocorrelation side lobes of pulsed signals, Geophysics 73 (3) (2008) Q19-Q27. doi:10.1190/1.2903820.

[3] M. Palmese, G. Bertolotto, A. Pescetto, A. Trucco, Experimental validation of a chirp-based underwater acoustic communication method, Proceedings of Meetings on Acoustics, Acoustical Society of America 4 (2008) 6 .

[4] P. D. Gupta, Workshop on gravitational waves and relativistic astrophysics, Indian Academy of Sciences - Journal of physics 63 (4) (2004) 877-882.

[5] M. Barbu, E. Kaminsky, R. Trahan, Fractional fourier transform for sonar signal processing, in: OCEANS, 2005. Proceedings of MTS/IEEE, 2005, pp. 1630 -1635 Vol. 2. doi:10.1109/OCEANS.2005.1639989.

[6] M. B. Charles E. Cook, Radar signals: An Introduction To Theory And Application, Academic Press, 1967.

[7] A. Springer, W. Gugler, M. Huemer, L. Reindl, C. Ruppel, R. Weigel, Spread spectrum communications using chirp signals, in: EUROCOMM 2000. Information Systems for Enhanced Public Safety and Security. IEEE/AFCEA, 2000, pp. 166 -170. doi:10.1109/EURCOM.2000.874794.

[8] M. Kowatsch, J. Lafferl, A spread-spectrum concept combining chirp modulation and pseudonoise coding, Communications, IEEE Transactions on 31 (10) (1983) 1133 - 1142. doi:10.1109/TCOM.1983.1095745.

[9] J. C. Curlander, R. N. McDonough, Synthetic Aperture Radar: Systems and Signal Processing, Wiley-Interscience, 1992.

[10] J. Xu, L. Durand, P. Pibarot, Nonlinear transient chirp signal modeling of the aortic and pulmonary components of the second heart sound, Biomedical Engineering, IEEE Transactions on 47 (10) (2000) 1328 1335. doi:10.1109/10.871405. 
[11] T. K. Gaisser, Cosmic Rays and Particle Physics, 1st Edition, Cambridge University Press, 1991.

[12] H. Tokuno, Y. Murano, S. Kawana, Y. Tameda, A. Taketa, D. Ikeda, S. Udo, S. Ogio, M. Fukushima, R. Azuma, On site calibration for new fluorescence detectors of the telescope array experiment, Nuclear Instruments and Methods in Physics Research Section A: Accelerators, Spectrometers, Detectors and Associated Equipment 601 (3) (2009) 364-371.

[13] M. A. B. Othman, C. Allen, J. Belz, D. Besson, B. Farhang-Boroujeny, D. Ikeda, I. Kravchenko, S. Kunwar, J. Lundquist, I. Myers, T. NakamuraA, H. Sagawa, P. Sokolsky, H. Takai, T. Terasawa, G. B. Thomson, T. T. A. Collaboration, Radar detection of uhecr air showers at the telescope array, in: 32nd International Cosmic Ray Conference, Beijing, 2011.

[14] J. D. Jackson, Classical Electrodynamics, 3rd Edition, Wiley, 1998.

[15] N. J. Willis, Bistatic Radar, 2nd Edition, SciTech Publishing, 2005.

[16] O. Machhi, N. Bershad, Adaptive recovery of a chirped sinusoid in noise. i. performance of the rls algorithm, Signal Processing, IEEE Transactions on 39 (3) (1991) 583 -594. doi:10.1109/78.80878.

[17] N. Bershad, O. Macchi, Adaptive recovery of a chirped sinusoid in noise. ii. performance of the lms algorithm, Signal Processing, IEEE Transactions on 39 (3) (1991) 595 -602. doi:10.1109/78.80879.

[18] P. Tichavsky, P. Handel, Two algorithms for adaptive retrieval of slowly time-varying multiple cisoids in noise, Signal Processing, IEEE Transactions on 43 (5) (1995) 1116 -1127. doi:10.1109/78.382397.

[19] P. Wei, J. Han, J. Zeidler, W. Ku, Comparative tracking performance of the lms and rls algorithms for chirped narrowband signal recovery, Signal Processing, IEEE Transactions on 50 (7) (2002) 1602-1609. doi:10.1109/TSP.2002.1011201.

[20] C. E. Cook, M. Bernfeld, Radar signals: an introduction to theory and application, 1st Edition, Academic Press, 1967. 
[21] S. M. Kay, Fundamentals of Statistical Signal Processing, Volume II: Detection Theory, 1st Edition, Prentice Hall, 1998.

[22] B. Chen, P. Willett, Detection of hidden markov model transient signals, Aerospace and Electronic Systems, IEEE Transactions on 36 (4) (2000) 1253 - 1268. doi:10.1109/7.892673.

[23] T. Luginbuhl, P. Willett, Tracking a general, frequency modulated signal in noise, in: Decision and Control, 1999. Proceedings of the 38th IEEE Conference on, Vol. 5, 1999, pp. 5076 -5081 vol.5. doi:10.1109/CDC.1999.833355.

[24] B. Carlson, E. Evans, S. Wilson, Search radar detection and track with the hough transform. i. system concept, Aerospace and Electronic Systems, IEEE Transactions on 30 (1) (1994) $102-108$. doi:10.1109/7.250410.

[25] J. Roger L. Easton, A. J. Ticknor, H. H. Barrett, Two-dimensional complex fourier transform via the radon transform, Appl. Opt. 24 (22) (1985) 3817-3824. doi:10.1364/AO.24.003817.

URL http://ao.osa.org/abstract.cfm?URI=ao-24-22-3817

[26] J. Xu, J. Yu, Y.-N. Peng, X.-G. Xia, Radon-fourier transform for radar target detection, i: Generalized doppler filter bank, Aerospace and Electronic Systems, IEEE Transactions on 47 (2) (2011) 1186-1202. doi:10.1109/TAES.2011.5751251.

[27] S. Kay, G. Boudreaux-Bartels, On the optimality of the wigner distribution for detection, in: Acoustics, Speech, and Signal Processing, IEEE International Conference on ICASSP '85., Vol. 10, 1985, pp. 1017-1020. doi:10.1109/ICASSP.1985.1168129.

[28] B. Boashash, N. A. Khan, T. Ben-Jabeur, Timefrequency features for pattern recognition using high-resolution tfds: A tutorial review, Digital Signal Processing 40 (2015) 1 - 30. doi:http://dx.doi.org/10.1016/j.dsp.2014.12.015.

[29] J. J. Healy, W. T. Rhodes, J. T. Sheridan, Cross terms of the wigner distribution function and aliasing in numerical simulations of paraxial optical systems, Optical Letters 35 (2010) 1142-1144. 
[30] S. Barbarossa, A. Zanalda, A combined wigner-ville and hough transform for cross-terms suppression and optimal detection and parameter estimation, in: Acoustics, Speech, and Signal Processing, 1992. ICASSP92., 1992 IEEE International Conference on, Vol. 5, 1992, pp. 173-176 vol.5. doi:10.1109/ICASSP.1992.226630.

[31] T. Gulum, A. Erdogan, T. Yildirim, P. Pace, A parameter extraction technique for fmcw radar signals using wigner-hough-radon transform, in: Radar Conference (RADAR), 2012 IEEE, 2012, pp. 0847-0852. doi:10.1109/RADAR.2012.6212255.

[32] B. Carlson, E. Evans, S. Wilson, Search radar detection and track with the hough transform. ii. detection statistics, Aerospace and Electronic Systems, IEEE Transactions on 30 (1) (1994) 109-115. doi:10.1109/7.250411.

[33] M. Othman, I. Myers, J. Belz, B. Farhang-Boroujeny, On radar detection of chirp signals with nondeterministic parameters in challenging noise background, in: Radar Conference (RADAR), 2013 IEEE, 2013, pp. 1-6. doi:10.1109/RADAR.2013.6585981.

[34] J. Illingworth, J. Kittler, A survey of the hough transform, Computer Vision, Graphics, and Image Processing 44 (1) (1988) 87 - 116.

[35] P. V. C. Hough, A method and means for recognizing complex patterns, U.S. Patent 3,069,654.

[36] S. H. Nawab, T. F. Quatieri, Advanced Topics in Signal Processing, Prentice-Hall, Inc., Upper Saddle River, NJ, USA, 1987.

URL http://dl.acm.org/citation. cfm?id=42739.42745

[37] A. Goldsmith, Wireless Communications, Cambridge University Press, New York, NY, USA, 2005.

[38] H. O. Lancaster, The Chi-squared Distribution, John Wiley and Sons Inc, 1969.

[39] I. Gradshteyn, I. Ryzhik, Table of Integrals, Series, and Products, 7th Edition, Academic Press, 2007. 
[40] J. Imberger, B. Boashash, Application of the wigner-ville distribution to temperature gradient microstructure: a new technique to study smallscale variations, J. of Physical Oceanography 16 (1986) 1997-2012. 